\title{
Revisiting the Extended Schmidt Law: The Important Role of Existing Stars in Regulating Star Formation
}

\author{
Yong Shi ${ }^{1,2}$ (1) , Lin Yan $^{3}$ (10), Lee Armus ${ }^{3}$, Qiusheng Gu${ }^{1,2}$ (1) , George Helou ${ }^{3}$, Keping Qiu ${ }^{1,2}$ (1), \\ Stephen Gwyn ${ }^{4}$, Sabrina Stierwalt ${ }^{5}\left(\mathbb{0}\right.$, Min Fang ${ }^{6}$ (i), Yanmei Chen ${ }^{1,2}$, Luwenjia Zhou ${ }^{1,2}$, Jingwen $\mathrm{Wu}^{7}{ }^{(1)}$, \\ Xianzhong Zheng ${ }^{8}$ (i), Zhi-Yu Zhang ${ }^{9}$ (i), Yu Gao ${ }^{8}$ (D) , and Junzhi Wang ${ }^{10}$ \\ ${ }^{1}$ School of Astronomy and Space Science, Nanjing University, Nanjing 210093, People's Republic of China; yshipku@gmail.com \\ ${ }^{2}$ Key Laboratory of Modern Astronomy and Astrophysics (Nanjing University), Ministry of Education, Nanjing 210093, People's Republic of China \\ ${ }_{3}^{3}$ Infrared Processing and Analysis Center, California Institute of Technology, 1200 E. California Boulevard, Pasadena, CA 91125, USA \\ ${ }^{4}$ NRC-Herzberg Astronomy and Astrophysics, National Research Council of Canada, 5071 West Saanich Road, Victoria, BC V9E 2E7, Canada \\ 5 Department of Astronomy, University of Virginia, P.O. Box 400325, Charlottesville, VA 22904, USA \\ ${ }^{6}$ Department Of Astronomy And Steward Observatory, University of Arizona, 933 N Cherry Avenue, Tucson, AZ 85721, USA \\ ${ }^{7}$ National Astronomical Observatories, Chinese Academy of Sciences, 20A Datun Road, Chaoyang District, Beijing, People's Republic of China \\ ${ }^{8}$ Purple Mountain Observatory, Chinese Academy of Sciences, 2 West Beijing Road, Nanjing 210008, People's Republic of China \\ ${ }^{9}$ Institute for Astronomy, University of Edinburgh, Royal Observatory, Blackford Hill, Edinburgh EH9 3HJ, UK \\ ${ }^{10}$ Shanghai Astronomical Observatory, Chinese Academy of Sciences, 80 Nandan Road, Shanghai 200030, People's Republic of China \\ Received 2017 August 8; revised 2017 December 15; accepted 2017 December 19; published 2018 February 1
}

\begin{abstract}
We revisit the proposed extended Schmidt law, which posits that the star formation efficiency in galaxies depends on the stellar mass surface density, by investigating spatially resolved star formation rates (SFRs), gas masses, and stellar masses of star formation regions in a vast range of galactic environments, from the outer disks of dwarf galaxies, to spiral disks and to merging galaxies, as well as individual molecular clouds in M33. We find that these regions are distributed in a tight power law as $\Sigma_{\mathrm{SFR}} \propto\left(\Sigma_{\mathrm{star}}^{0.5} \Sigma_{\mathrm{gas}}\right)^{1.09}$, which is also valid for the integrated measurements of disk and merging galaxies at high-z. Interestingly, we show that star formation regions in the outer disks of dwarf galaxies with $\Sigma_{\mathrm{SFR}}$ down to $10^{-5} M_{\odot} \mathrm{yr}^{-1} \mathrm{kpc}^{-2}$, which are outliers of both the KennicuttSchmidt and Silk-Elmegreen laws, also follow the extended Schmidt law. Other outliers in the Kennicutt-Schmidt law, such as extremely metal-poor star formation regions, also show significantly reduced deviation from the extended Schmidt law. These results suggest an important role for existing stars in helping to regulate star formation through the effect of their gravity on the midplane pressure in a wide range of galactic environments.
\end{abstract}

Key words: galaxies: evolution - galaxies: starburst - ISM: atoms - ISM: molecules - stars: formation

\section{Introduction}

Stars are born in clouds of cold gas. Understanding how gas is converted into stars is crucial for constraining theories of galaxy formation and evolution across cosmic time. In the early universe, primordial gas with little or no metals collapsed into first- and second-generation stars whose radiation is responsible for re-ionizing the universe. The subsequent rise in the cosmic star formation rate (SFR) density until $z \sim 2$, followed by the decline to $z=0$, has been well mapped in a broad sense by modern, multi-wavelength surveys (e.g., Madau \& Dickinson 2014). The driving mechanism of this cosmic SFR evolution is thought to be related to the cold gas reservoir available within and surrounding galaxies, and the physical conditions that allow this gas to be converted into new stars (Genzel et al. 2010; Tacconi et al. 2013).

Empirical relationships between the SFR and cold gas mass (or star formation law) as first proposed by Schmidt (1959) are powerful tools for understanding star formation and also useful prescriptions for creating stars from cold gas in cosmological simulations. Since the pioneering work of Kennicutt (1989), studies have established the power-law form of the relationship between the SFR surface density $\left(\Sigma_{\mathrm{gas}}\right)$ and gas (atomic + molecular) mass surface density ( $\left.\Sigma_{\text {gas }}\right)$, i.e., the KennicuttSchmidt law, in the form $\Sigma_{\mathrm{SFR}} \propto \Sigma_{\text {gas }}^{N}$, with $N$ around 1.4. It is found that for star formation regions in individual galaxies, the relationship shows variations in both slope and normalization (Kennicutt et al. 2007; Leroy et al. 2008; Boquien et al. 2011;
Shi et al. 2011), but for ensembles of galaxies covering a large dynamic range of SFRs, the relationship has a relatively small dispersion of about 0.3 dex (Kennicutt 1998a; Wyder et al. 2009; Shi et al. 2011). This suggests that the gas mass surface density is the primary factor in regulating the SFR surface density.

Deviations from the Kennicutt-Schmidt law occur at the low density end $\left(\Sigma_{\text {gas }} \lesssim 10 M_{\odot} \mathrm{pc}^{-2}\right.$ or $\left.\Sigma_{\mathrm{SFR}} \lesssim 10^{-3} M_{\odot} \mathrm{yr}^{-1} \mathrm{kpc}^{-2}\right)$ where star formation is systematically below the power-law extrapolation from the higher gas density regime, including lowsurface-brightness (LSB) galaxies and the outer disks of galaxies (Kennicutt 1998a; Bigiel et al. 2008). Such deviation implies an additional parameter besides the gas mass that may help to regulate ongoing star formation. At least two modified versions of the Schmidt relationship have been proposed. One is the so-called Silk-Elmegreen relationship that invokes the dynamical orbital timescale as $\Sigma_{\mathrm{SFR}} \propto \Sigma_{\mathrm{gas}} / t_{\mathrm{dyn}}$ (Elmegreen 1997; Silk 1997). Another is the extended Schmidt law that invokes the stellar mass surface density as $\Sigma_{\mathrm{SFR}} \propto \Sigma_{\mathrm{gas}} \Sigma_{\text {star }}^{N}$, with $N$ around 0.5 (Shi et al. 2011). In both relationships, the LSB galaxies and outer disks of spiral galaxies, along with the main disks of spirals and starburst galaxies, define a power law across the whole dynamic range, with no obvious break at the low density end down to $\Sigma_{\mathrm{SFR}} \sim 10^{-4} M_{\odot} \mathrm{yr}^{-1} \mathrm{kpc}^{-2}$. In this study, we will include measurements of star formation regions in the outer disks of dwarf galaxies with $\Sigma_{\text {SFR }} 10$ times lower than LSB galaxies and outer disks of spirals to further test these two alternative models, and thus to establish which parameter (dynamical timescale versus 
Table 1

The CFHT Observations of Three Dwarf Galaxies

\begin{tabular}{llccc}
\hline \hline Object & Band & Observing Date & $\begin{array}{c}\text { Exposure Time } \\
(\mathrm{s})\end{array}$ & $\begin{array}{c}\text { Seeing } \\
\left({ }^{\prime \prime}\right)\end{array}$ \\
\hline Ho II & $g$-band & 2014 Feb 24 & 2076 & 0.79 \\
& $r$-band & 2014 Feb 24 & 2415 & 0.62 \\
\multirow{2}{*}{ Ho I } & $g$-band & 2014 Mar 27 & 2768 & 0.84 \\
& $r$-band & 2014 Mar 28-Apr 4 & 2760 & 0.63 \\
\multirow{2}{*}{ DDO 154 } & $g$-band & 2014 Jul 2 & 2768 & 0.78 \\
& $r$-band & 2014 Jul 2 & 2415 & 0.65 \\
\hline
\end{tabular}

stellar mass) in addition to the gas mass plays a more important secondary role in regulating star formation.

In addition, LSB galaxies and galaxy outer disks, deviations from the Kennicutt-Schmidt law are also seen for other types of galaxies. For example, individual star formation regions in extremely metal-poor galaxies with metallicities below $10 \%$ of solar show significantly lower SFRs than those in spiral galaxies at given gas mass densities in the Kennicutt-Schmidt plane (e.g., Shi et al. 2014). The disk-averaged SFRs of lowmass metal-poor galaxies also deviate from the KennicuttSchmidt law (e.g., Roychowdhury et al. 2015). Besides, radio galaxies with jets are shown to have lower SFRs than those predicted by the Kennicutt-Schmidt law (Lanz et al. 2016). In this study, we will examine if these galaxies show reduced deviations from the extended Schmidt law upon including the stellar mass term.

With the spatially resolved observations of nearby galaxies as enabled by multi-wavelength modern facilities, we can also evaluate the star formation law on sub-kiloparsec scales in different galactic environments. Such measurements relate the local SFRs to in situ gas and stellar mass that may be physically related to current star formation. The paper is organized as follows. In Section 2 we present the data and measurements of physical parameters, including stellar mass, gas mass, and SFR surface density. The updated version of the extended Schmidt law is presented in Section 3. In Section 4, we discuss the distribution of the outliers of the Kennicutt-Schmidt law in the extended Schmidt plane. The origin of the extended Schmidt law is discussed in Section 5. The conclusion is present in Section 6.

\section{Data and Measurements of Physical Parameters}

\subsection{Outer Disks of Three Dwarf Galaxies}

To test the role of the stellar mass in regions of extremely low $\Sigma_{\mathrm{SFR}}$, we selected three dwarf galaxies (Ho II, Ho I, and DDO 154) that have deep H I data as observed in the program of the H I Nearby Galaxy Sample (Walter et al. 2008), as well as deep far-UV images from the GALEX data archive. The H I images were naturally weighted with a beam size of $\sim 14^{\prime \prime} \times 12$ ". 5 and a typical column density sensitivity of $9 \times 10^{19} \mathrm{~cm}^{-2}$ at this resolution. For these three galaxies, single-dish measurements indicate that there is no significant missing $\mathrm{H}$ I flux at this resolution (Walter et al. 2008). The GALEX image has a spatial resolution of about $5^{\prime \prime}$ (Morrissey et al. 2007). The archive-produced sky image was subtracted from the target image, with the $1 \sigma$ scatter around (1-2) $\times$ $10^{-8} \mathrm{Jy}$ at the pixel scale of $1 . \prime 5$, corresponding to a SFR surface density of $(1-2) \times 10^{-5} M_{\odot} \mathrm{yr}^{-1} \mathrm{kpc}^{-2}$.
The optical observations of the above three dwarf galaxies were carried out at the $g$ - and $r$-bands with the MegaCAM of CFHT (PI: Y. Shi) through the China Telescope Access Program. The observing details are listed in Table 1, with typical seeing of $0 . \prime 8$ in the $g$-band and $0 . " 6$ in the $r$-band. To best recover the faint outer disk emission of our galaxies, the observations were dithered using the LDP-CCD-7 mode as developed by the CFHT Elixir team. The dither uses large offsets $\left(\sim 15^{\prime}\right)$ to move the target across the MegaCAM mosaic such that the underlying background of the target out to the dither size can be recovered and subtracted from the series of exposures. The outer disks of our three galaxies extend out to $5^{\prime}-10^{\prime}$, which is smaller than the dither size so that the full advantage of the above dither pattern can be fulfilled. Individual frames were first reduced with Elixir pipeline (Magnier \& Cuillandre 2004) including the bias subtraction, the flat-field response correction. and the application of the relative flux calibration. Then the individual frames were mosaicked by taking care of diffuse light using the MegaPipe image processing pipeline (Gwyn 2008) that includes the image grouping, astrometric calibration, photometric calibration, image stacking, and catalog generation.

To probe star-forming regions with $\Sigma_{\text {SFR }}$ below $10^{-4} M_{\odot} \mathrm{yr}^{-1} \mathrm{kpc}^{-2}$, where the previous study has not yet probed (Shi et al. 2011), we defined the outer disks as being between two contours at the SFR surface densities of $10^{-4}$ and $10^{-5} M_{\odot} \mathrm{yr}^{-1} \mathrm{kpc}^{-2}$, respectively. We then filled the outer disk with individual circle regions of $30^{\prime \prime}$ as outer disk star-forming regions, as shown in Figure 1. In total 74, 25, and 15 regions are identified for Ho II, Ho I, and DDO 154, respectively.

The surface densities of SFR, gas mass, and stellar mass are measured with the following procedure. As stated above, the archived produced background image is subtracted from the target image, based on which far-UV surface brightness is converted to the SFR surface density with a Kroupa initial mass function (IMF) using the following formula (Leroy et al. 2008):

$$
\Sigma_{\mathrm{SFR}}=8.1 \times 10^{-2} \cos (i) I_{\mathrm{FUV}},
$$

where $\Sigma_{\mathrm{SFR}}$ is in $M_{\odot} \mathrm{yr}^{-1} \mathrm{kpc}^{-2}, I_{\mathrm{FUV}}$ is in $\mathrm{MJy} \mathrm{sr}^{-1}$, and $i$ is the inclination angle of the galaxy measured at $R_{25}$. Note that the H I warp is seen in DDO 154 but all of our outer disk starforming regions are within the radius where the warp occurs (Carignan \& Beaulieu 1989). No warps are seen in the remaining two galaxies (Ho I and Ho II; Holwerda et al. 2011). As a result, the adopted inclination at $R_{25}$ is a reasonable value to use for the $\mathrm{H}$ I surface density estimate. No dust extinction is assumed for these outer disk regions. The associated uncertainty is the quadratic sum of the photometric uncertainty plus the systematic uncertainty of $0.3 \mathrm{dex}$.

The total gas mass of each star-forming region is the $\mathrm{H} \mathrm{I}$ gas mass multiplied by a factor of 1.36 to account for helium following the formula (Walter et al. 2008):

$$
\Sigma_{\text {atomic-gas }}=1.20 \times 10^{4} \cos (i)(1+z)^{3} \frac{\operatorname{arcsec}^{2}}{\text { bmaj } \times \operatorname{bmin}} S_{\mathrm{H}_{\mathrm{I}}} \Delta v,
$$

where $i$ is the inclination angle of the galaxy, $z$ is the redshift of the galaxy, $\Sigma_{\text {atomic-gas }}$ is in $M_{\odot} \mathrm{pc}^{-2}, \quad S_{\mathrm{H}} \Delta v$ is in $\mathrm{Jy} \mathrm{km} \mathrm{s}^{-1}$ beam $^{-1}, b_{\text {maj }}$ and $b_{\text {min }}$ are the major and minor beam sizes in arcsec, respectively. Here, we assumed no molecular 
Holl

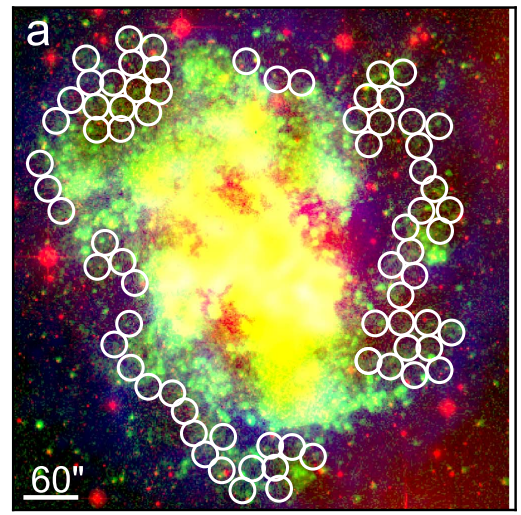

$\mathrm{Hol}$

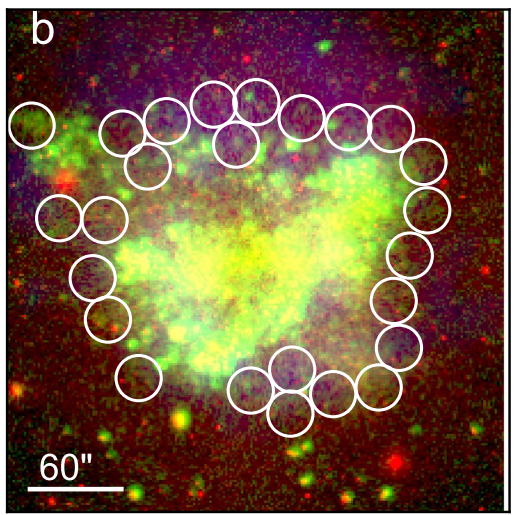

DDO 154

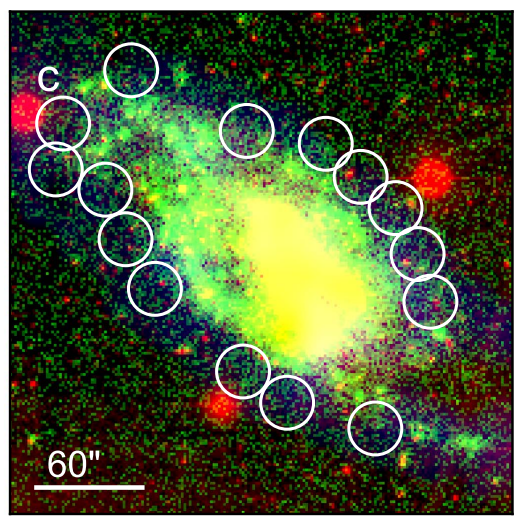

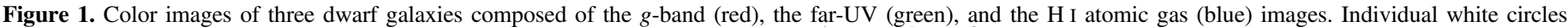
indicate star-forming regions in the outer disks with low SFR surface density $\left(<10^{-4} M_{\odot} \mathrm{yr}^{-1} \mathrm{kpc}^{-2}\right)$.

gas in the outer disk regions. The error is assumed to be $0.2 \mathrm{dex}$ to account for the flux calibration uncertainty.

To derive the stellar mass, we first measured the $g$ - and $r$-band photometry by summing the fluxes of individual detected sources within each $30^{\prime \prime}$ aperture based on the catalog produced by MegaPipe. Here, the high spatial resolution (0."6-0."8), combined with deep exposures, enable us to resolve individual sources within the aperture of each region. For one field (DDO 154) covered by the Sloan Digital Sky Survey, we compared the MegaPipe photometry of all point sources in the one square degree field to those by SDSS and found no systematic difference $(<0.02 \mathrm{mag})$. We also performed aperture photometry by summing all photons within the aperture of each region in the image after subtracting the global sky background, and performed a similar measurement but after subtracting both global and local backgrounds. These two methods give similar distributions of the stellar masses overall, compared to the first method. This indicates that the light within the aperture is dominated by the point sources, causing all the methods to give similar results. We adopted the results using the first method, which is not subject to the uncertainty caused by the global sky background subtraction over a large field of view and thus has a higher photometric accuracy.

To obtain the stellar mass from the $g$ and $r$ photometry, we used the Sloan MPA/JHU catalog (Brinchmann et al. 2004) to obtain the dependence of the mass-to-light ratio at the $r$-band on the $g-r$ color:

$$
\log M_{\text {star }}+M_{r \text {-band }} / 2.5=1.13+1.49(g-r),
$$

where $M_{\text {star }}$ is the stellar mass in $M_{\odot}$, and $M_{r \text {-band }}$ is the $r$-band absolute AB magnitude. As the $g-r$ color of the majority of the SDSS galaxies lies between 0.1 and 0.9 , for outer disk regions with $g-r>0.9$, we fixed the mass-to-light ratio to that for a population with $g-r=0.9$. Similarly, the mass-to-light ratio of regions with $g-r<0.1$ was fixed to that for a population with $g-r=0.1$. For about $60 \%$ of the regions with $g-r$ between 0.1 and 0.9 , the mass-to-light ratio has a scatter of $0.25 \mathrm{dex}$ and the remaining $40 \%$ outside the range is assumed to have a scatter of 0.5 dex. The final error of the stellar mass, mainly between 0.25 and $0.5 \mathrm{dex}$, includes the photometric error in the $g$ - and $r$-bands, as well as the error in the mass-to-light ratio. Our SDSS-based mass-to-light ratio in the $r$-band as a function of $g-r$ color is quite consistent with the result derived from the 50,000 stellar population synthesis models (Zibetti et al. 2009), with a difference of less than 0.1 dex for $g-r$ between 0.1 and 0.9 .

The orbital dynamical time of Ho II and DDO 154 is based on the H I rotation curve available in Oh et al. (2015).

\subsection{Local Spiral Galaxies}

The spatially resolved measurements of SFRs, gas masses, and stellar masses of 12 spiral galaxies are carried out following the literature (Bigiel et al. 2008; Shi et al. 2011). The aperture size is set to be $750 \times 750 \mathrm{pc}^{2}$, which can be achieved by the angular resolution of the data at the largest distance of the sample. The SFRs are combinations of the farUV data from the GALEX archive and the infrared data from the SIRTF Nearby Galaxies Survey (Kennicutt et al. 2003), based on the equation (Leroy et al. 2008)

$$
\Sigma_{\mathrm{SFR}}=8.1 \times 10^{-2} \cos (i) I_{\mathrm{FUV}}+3.2 \times 10^{-3} \cos (i) I_{24 \mu \mathrm{m}},
$$

where $i$ is the inclination angle of the galaxy, $\Sigma_{\mathrm{SFR}}$ is in $M_{\odot} \mathrm{yr}^{-1} \mathrm{kpc}^{-2}$, and both $I_{\mathrm{FUV}}$ and $I_{24 \mu \mathrm{m}}$ are in $\mathrm{MJy} \mathrm{sr}^{-1}$. The $\mathrm{H}$ I data are from the H I Nearby Galaxy Survey (Walter et al. 2008) and CO data are carried out by the BIMA SONG CO $J=1-0$ map (Helfer et al. 2003), from which the total gas is derived as $\Sigma_{\text {gas }}=\Sigma_{\text {atomic-gas }}+\Sigma_{\text {mol-gas. }}$ The atomic gas mass follows Equation (1). The molecular part is based on

$$
\begin{aligned}
\Sigma_{\text {mol-gas }}= & 393 \cos (i) \frac{\operatorname{arcsec}^{2}}{\mathrm{bmaj}^{2} \mathrm{bmin}} \\
& \times \frac{X_{\mathrm{CO}}}{\left(2 \times 10^{20} \frac{\mathrm{cm}^{-2}}{\mathrm{~K} \mathrm{~km} \mathrm{~s}^{-1}}\right)}(1+z)^{3} S_{\mathrm{CO}} \Delta v,
\end{aligned}
$$

where $i$ is the inclination angle of the galaxy, $\Sigma_{\text {mol-gas }}$ is in $M_{\odot} \mathrm{pc}^{-2}$, and $S_{\mathrm{CO}} \Delta v$ is in $\mathrm{Jy} \mathrm{km} \mathrm{s}^{-1}$ beam ${ }^{-1}$. Here, a factor of 1.36 has been included to account for the helium. The CO emission is $J=1-0$ and we adopt $X_{\mathrm{CO}}=2 \times 10^{20} \mathrm{~cm}^{-2}$ for spiral galaxies.

Based on the available Spitzer $3.6 \mu \mathrm{m}$ data (Kennicutt et al. 2003), the stellar mass is derived by the formula (Leroy 
et al. 2008)

$$
\Sigma_{\text {star }}=140 \cos (i) I_{2 \text { mass- } \mathrm{K}}=280 \cos (i) I_{3.6 \mu \mathrm{m}},
$$

where $i$ is the inclination angle of the galaxy, $\Sigma_{\text {star }}$ is in $M_{\odot} \mathrm{pc}^{-2}$, and both $I_{2 \mathrm{mass}-\mathrm{K}}$ and $I_{3.6 \mu \mathrm{m}}$ are in $\mathrm{MJy} \mathrm{sr}^{-1}$. For five galaxies that have SDSS optical images, we also derived the stellar mass using Equation (2) and found that the difference from the IRAC-based one is smaller than $0.2 \mathrm{dex}$.

\subsection{Luminous Infrared Galaxies in the Local Universe}

NGC 1614-NGC 1614 is a luminous infrared galaxy (LIRG) with an infrared luminosity of $10^{11.65} L_{\odot}$ (Armus et al. 2009) at the late stage of a merging process. It likely has experienced a minor merging event that triggered intense star formation in a circumnuclear ring region. We carried out spatially resolved measurements for the nuclear region, as well as individual regions in the star-forming ring of NGC 1614. The aperture size is about $100-200 \mathrm{pc}$, which is about two times the angular resolution.

The total gas mass is measured based on the CO 6-5 map as observed by ALMA (Xu et al. 2015), with a synthesized beam of $0.26 \times 0.20 \operatorname{arcsec}^{2}$. We retrieved the data from the ALMA archive and obtained the intensity map by integrating the velocity from 4500 to $5000 \mathrm{~km} \mathrm{~s}^{-1}$. To estimate the molecular gas mass from $\mathrm{CO} 6-5$, we used the formula in the literature (Xu et al. 2015) with a slightly smaller $X_{\mathrm{CO}}$ : CO 6-5 is first converted to $\mathrm{CO} 2-1$ by assuming a brightness temperature ratio of 0.72 between the two; to derive the factor from $\mathrm{CO} 2-1$ to the molecular gas mass, we converted the $\mathrm{CO}$ (2-1) flux (König et al. 2013) to CO (1-0) by dividing a factor of $3.1(0.78$ in the brightness temperature) (Wilson et al. 2008) and then used the conversion factor $X_{\mathrm{CO}}=2 \times 10^{20} \mathrm{~cm}^{-2}$ for CO 1-0 with an additional factor of 1.36 for the helium. The final formula is

$$
\Sigma_{\mathrm{gas}}=13.5 \times f_{\mathrm{CO}(6-5)},
$$

where $\Sigma_{\text {gas }}$ is in $M_{\odot} \mathrm{pc}^{-2}$ and $f_{\mathrm{CO}(6-5)}$ is in $\mathrm{Jy} \mathrm{km} \mathrm{s}^{-1} \operatorname{arcsec}^{-2}$.

SFRs are measured based on the radio continuum map. We retrieved radio $8.4 \mathrm{GHz}$ ( $X$-band) and $4.86 \mathrm{GHz}$ ( $C$-band) intensity maps from the VLA archive that have synthesized beam sizes of $0.26 \times 0.21$ and $0.60 \times 0.40 \operatorname{arcsec}^{2}$, respectively. With a Kroupa IMF, the SFR is based on the formula (Murphy et al. 2012)

$$
\mathrm{SFR}=\frac{10^{-27}}{2.18\left(\frac{T_{\mathrm{e}}}{10^{4} \mathrm{~K}}\right)^{0.45}\left(\frac{\nu}{\mathrm{GHz}}\right)^{-0.1}+15.1\left(\frac{\nu}{\mathrm{GHz}}\right)^{-\alpha^{\mathrm{NT}}}} L_{\nu},
$$

where SFR is in $M_{\odot} \mathrm{yr}^{-1}, T_{\mathrm{e}}$ is the thermal electron temperature at $10^{4} \mathrm{~K}, \alpha^{\mathrm{NT}}$ is the non-thermal radio spectral index with a suggested value of 0.85 , and $L_{\nu}$ is in $\operatorname{erg~s}^{-1} \mathrm{~Hz}^{-1}$. With $\alpha^{\mathrm{NT}}=0.85$, the two SFRs based on the $X$-band and $C$-band radio data are quite consistent with each other, with a median offset of 0.12 dex. The final result is the mean value of the two SFRs. Note that because of the different formulae used in the literature (Xu et al. 2015), our SFRs are about a factor of two lower.

To measure the stellar mass, we retrieved the archived images of the Hubble Space Telescope in the filters of ACSF435W, ACS-F814W, NICMOS-F110W, NICMOS-F160W, and NICMOS-F222M, whose spatial resolutions are comparable to those of $\mathrm{CO} 6-5$ and radio continuum images. The astrometry of each NICMOS image is first registered to that of ACS by matching several field stars. Then all HST images are further registered by matching the optical center to the radio center. The broadband SED within each aperture is then constructed and fitted to derive the stellar synthesis model (Brinchmann et al. 2004).

IC 4687-IC 4687 is an LIRG with an IR luminosity of about $10^{11.3} L_{\odot} \quad($ Pereira-Santaella et al. 2011). Spatially resolved measurements of SFRs and gas masses of IC 4687 were carried out in Pereira-Santaella et al. (2016). The SFRs are based on the extinction-corrected $\mathrm{Pa} \alpha$ emission as observed by $H S T$, and the gas mass is based on $\mathrm{CO} J=2-1$ emission as observed by ALMA. The conversion factor is taken to be $X_{\mathrm{CO}}=2 \times 10^{20} \mathrm{~cm}^{-2}$, as argued in Pereira-Santaella et al. (2011). The individual region has a diameter of 0 !' 35 . We have retrieved ACS-F435W, ACS-F814W, NICMOS-F110W, and NICMOS-F160W archive data from HST. Following procedures similar to those mentioned above, the optical/NIR SED is constructed for each region and the stellar mass is estimated through the stellar synthesis model (Brinchmann et al. 2004).

(U)LIRG at $1^{\prime \prime}$ resolution. A small sample of six local (U) LIRGs have CO J 3-2 and $880 \mu \mathrm{m}$ images as observed with the Submillimeter Array (SMA; Wilson et al. 2008). We retrieved the raw data from the SMA archive and re-reduced them.

For each galaxy, an aperture of about $1 \mathrm{kpc}$ is defined to enclose the whole extent of $\mathrm{CO}$ emission, and the integrated $\mathrm{CO} J$ 3-2 flux is first converted to $\mathrm{CO} J 1-0$ by assuming a ratio $\mathrm{CO}(J=3-2) / \mathrm{CO}(J=1-0)=0.5$ in the brightness temperature (Wilson et al. 2008), then converted to the molecular gas with $X_{\mathrm{CO}}=2.0 \times 10^{20} \mathrm{~cm}^{-2}$, with an additional factor of 1.36 to include the helium

$$
M_{\mathrm{mol}}=8.7 L_{\mathrm{CO}}^{\prime}(3-2),
$$

where $L_{\mathrm{CO}}^{\prime}(3-2)=3.2546 \times 10^{7}\left(\frac{\mathrm{S}_{\mathrm{CO}}}{\mathrm{Jy} \mathrm{km} \mathrm{s}^{-1}}\right) \quad\left(\frac{D_{\mathrm{L}}}{\mathrm{Mpc}}\right)^{2}$ $\left(\frac{\nu_{0}}{\mathrm{GHz}}\right)^{-2}(1+z)^{-1}$. The total $880 \mu \mathrm{m}$ flux within the defined aperture is converted to the far-IR luminosity by multiplying by a factor of 5000 for an infrared template at $\log \left(L_{\mathrm{TIR}} / L_{\odot}\right)=$ 11.7 (Rieke et al. 2009). Then it is coverted to the SFR based on the formula (Kennicutt 1998b) with a Kroupa IMF:

$$
\frac{\mathrm{SFR}}{\mathrm{M}_{\odot} \mathrm{yr}^{-1}}=3.0 \times 10^{-44} \frac{L_{\mathrm{TIR}}}{\mathrm{erg} \mathrm{s}^{-1}} \text {. }
$$

Similar to NGC 1614, the reduced HST images were retrieved from the archive at ACS-F435W, ACS-F814W, NICMOS-F110W, NICMOS-F160W, and NICMOS-F222M. Here, the NICMOS image is registered to the ACS image using the field stars. No further comparison in the astrometry between ACS and SMA images was done, as the accuracy $(\sim 0$ ! 1$)$ is high enough given the adopted apertures of $1^{\prime \prime}-2^{\prime \prime}$. The stellar mass is again based on the SED fitting (Brinchmann et al. 2004) to the broad SED of HST photometry.

\subsection{Giant Molecular Clouds (GMCs) In M33}

M33 was mapped with IRAM $30 \mathrm{~m}$ at CO (2-1) mainly along the major axis, with a coverage of $650 \operatorname{arcmin}^{2}$ at a spatial resolution of $12^{\prime \prime}$ (Gratier et al. 2010). Circle apertures were defined to enclose individual $\mathrm{CO}$ peaks, with a diameter of $18^{\prime \prime}$ corresponding to a physical scale of $76 \mathrm{pc}$. The CO $J=2-1$ is converted to $\mathrm{CO} J=1-0$ with a ratio of 0.7 in the brightness temperature and to the total molecular gas based on 
Table 2

The Integrated Properties of High- $z$ Starbursts

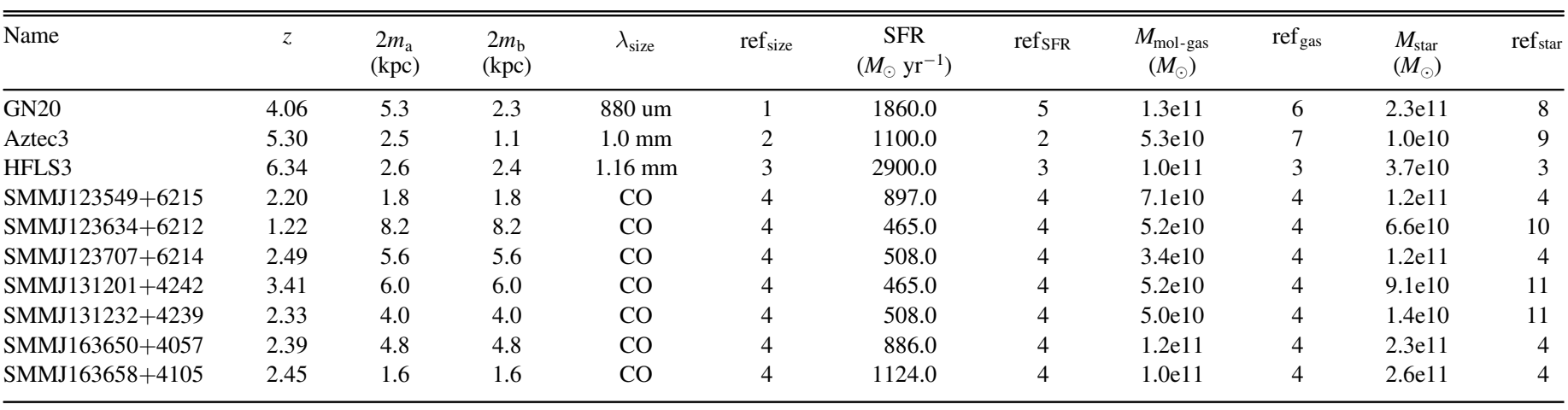

Note. (1) Hodge et al. (2015), (2) Riechers et al. (2014). (3) Riechers et al. (2013), (4) Genzel et al. (2010), (5) Hodge et al. (2015), (6) Carilli et al. (2011), (7) Riechers et al. (2010), (8) Daddi et al. (2009), (9) Capak et al. (2011), (10) Hainline et al. (2011), (11) Hainline et al. (2011).
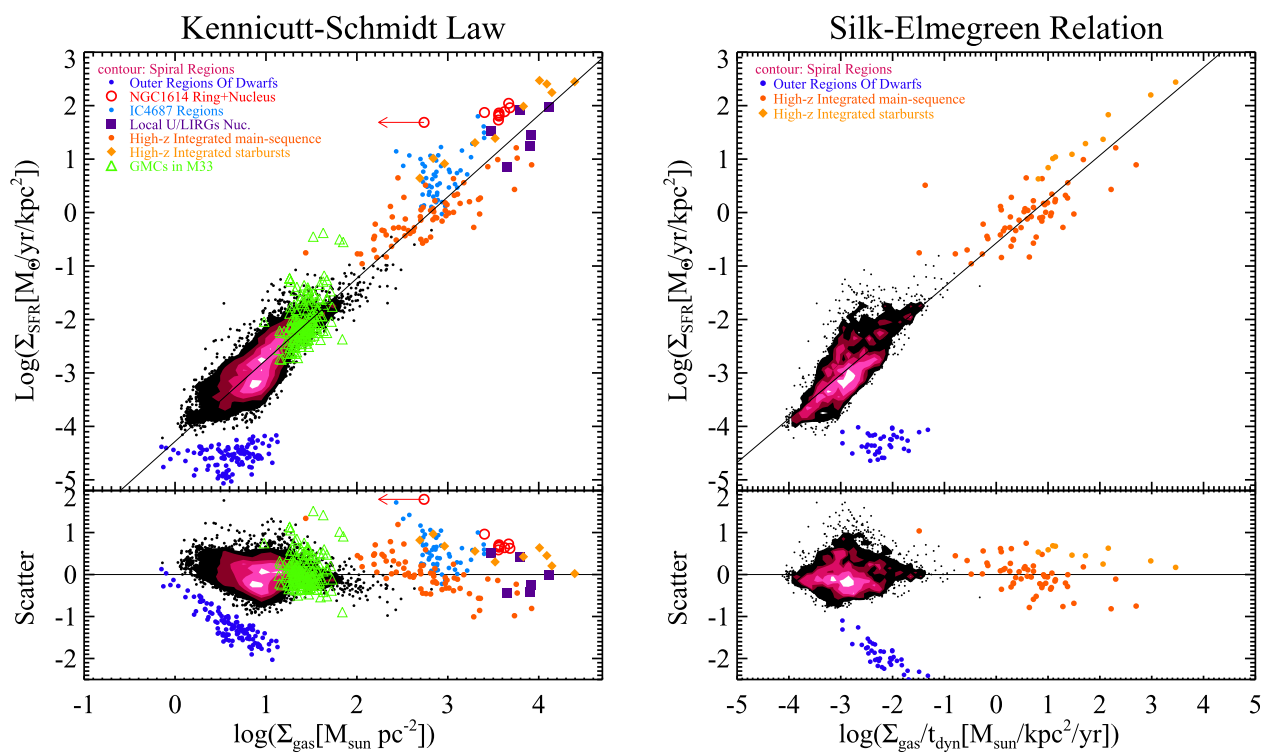

Figure 2. Kennicutt-Schmidt law (left top) and associated scatter (left bottom); the Silk-Elmegreen law (right top) and associated scatter (right bottom). The solid lines in all panels are the best fits. For the data of each sub-sample, please see the text.

Equation (5). The H I map was also available (Gratier et al. 2010) at a resolution of 17"; its emission is converted to the gas mass based on Equation (2). The SFR is based on the combination of Spitzer $24 \mu \mathrm{m}$ images (Tabatabaei et al. 2007) and GALEX far-UV images, following Equation (4), while the stellar mass is based on the Spitzer $3.6 \mu \mathrm{m}$ image (Dale et al. 2009), following Equation (6). Although M33 has a warped HI disk (Rogstad et al. 1976; Deul \& van der Hulst 1987), all our regions are within the radius where warp occurs and thus our measurements should not be affected by the warp.

\subsection{High-z Star-forming Galaxies and Starburst Galaxies}

We compiled a sample of high- $z$ star-forming and starburst galaxies, and adopted the integrated SFRs, gas masses, and stellar masses as measured in the literature. The half-light radius of the gas distribution or optical image is used to calculate the surface density with the formula $\Sigma=0.5 \mathrm{M} /$ $\pi / r_{0.5}^{2} . X_{\mathrm{CO} J=1-0}=2.0 \times 10^{20} \mathrm{~cm}^{-2}$ is used for both starforming and starburst galaxies. All star-forming galaxies in the literature (Tacconi et al. 2013) with available half-light radii are included here. The high- $z$ starburst galaxies are based on several works from the literature, as listed in Table 2.

\section{An Updated Version of the Extended Schmidt Law}

We present the Kennicutt-Schmidt law, the Silk-Elmegreen law, and the extended Schmidt law in the left panel of Figure 2, and the right panels of Figures 2 and 3, respectively. The data includes star formation regions in outer disks of dwarfs that are selected to have $\Sigma_{\mathrm{SFR}}$ below $10^{-4} M_{\odot} \mathrm{yr}^{-1} \mathrm{kpc}^{-2}, 750 \mathrm{pc}$ grids of spiral galaxies from the main disks to the outer disks, individual GMCs in M33, individual star formation regions in LIRGs NGC 1614 and IC 4687 as observed by ALMA, nuclear regions of local ULIRGs as observed by SMA, and high- $z$ integrated main-sequence and starburst galaxies. Only part of the above galaxies had rotation curves available in the literature to be included in the Silk-Elmegreen plot.

As found by Shi et al. (2011), the main and outer disks of spiral disks, nearby (L)ULIRGs, and high- $z$ star-forming/ starburst galaxies follow a well-defined sequence in the extended Schmidt plane, along with two additional data sets included in this study, which are regions in the outer disks of 


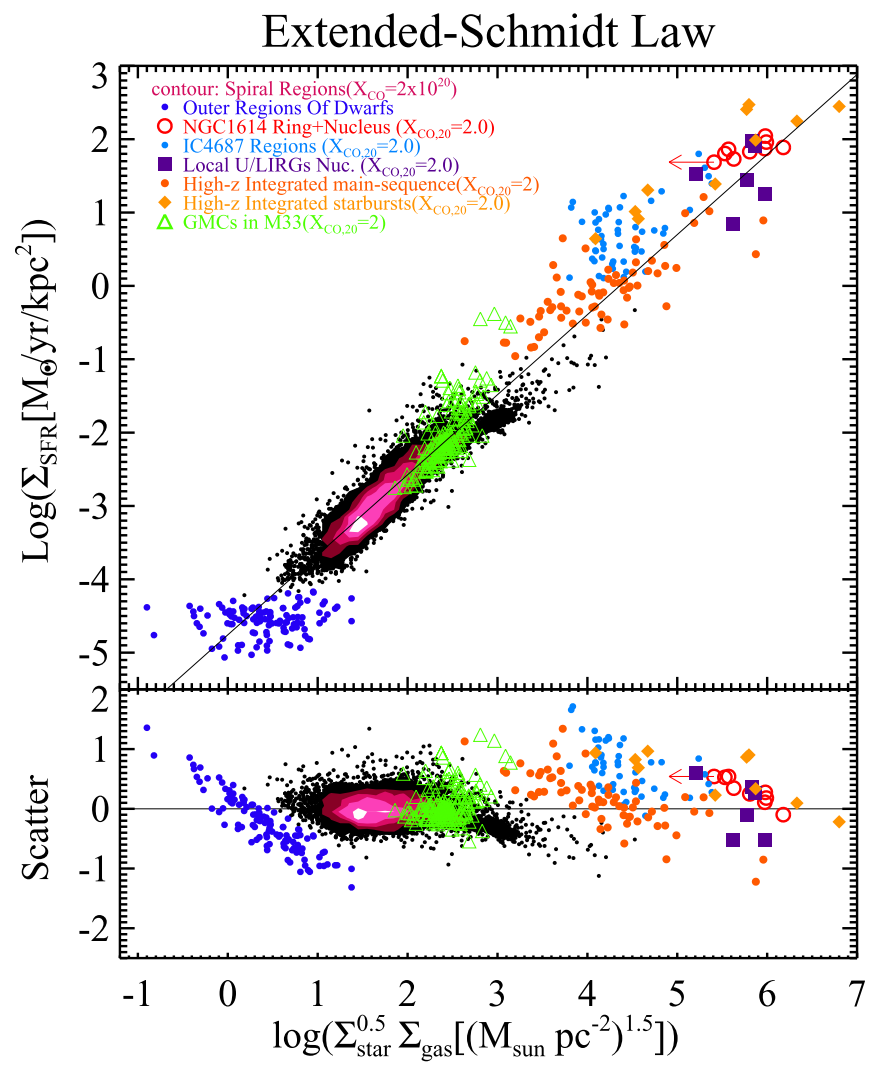

Figure 3. Extended Schmidt law (top panel) and the associated scatter (bottom panel) where the solid line is the best fit.

dwarf galaxies and individual GMCs in M33. The slope of the best-fitted extended Schmidt law is slightly super-linear:

$$
\Sigma_{\mathrm{SFR}}=10^{-4.76}\left(\Sigma_{\mathrm{star}}^{0.5} \Sigma_{\mathrm{gas}}\right)^{1.09}
$$

where $\Sigma_{\mathrm{SFR}}$ is in $M_{\odot} \mathrm{yr}^{-1} \mathrm{kpc}^{-2}$, and both $\Sigma_{\text {star }}$ and $\Sigma_{\text {gas }}$ are in $M_{\odot} \mathrm{pc}^{-2}$. The $1 \sigma$ apparent scatter of the relationship is about 0.30 dex. Table 3 lists the median offsets and standard deviations from the best fit of three relationships for galaxies of different types. The associated error bars were derived based on the bootstrap. As shown in the table, the outer disks of dwarf galaxies show much larger median offsets in the Kennicutt-Schmidt and Silk-Elmegreen laws than in the extended Schmidt law. Local spirals have no offsets in all three relationships but have the smallest scatters in the extended Schmidt law. GMCs in M33 have almost zero offsets in both the Kennicutt-Schmidt and extended Schmidt laws, but show a smaller scatter in the latter, which is also the case for high- $z$ galaxies. Local (U)LIRGs show similar systematic offsets and scatter in both extended Schmidt and KennicuttSchmidt laws.

Star formation regions in the outer disks of dwarf galaxies follow the extended Schmidt law well, but deviate significantly from the Kennicutt-Schmidt law and Silk-Elmegreen law. The three dwarf galaxies presented here allow us to investigate extremely low $\Sigma_{\mathrm{SFR}}$ down to $10^{-5} M_{\odot} \mathrm{yr}^{-1} \mathrm{kpc}^{-2}$. These regions are known to pose a challenge not only to the Kennicutt-Schmidt law (Kennicutt 1998a; Bigiel et al. 2010) but also to the Silk-Elmegreen law (Elmegreen 1997; Silk 1997; Kennicutt 1998a; Roychowdhury et al. 2015). Dwarf galaxies are small, thus their orbital timescale is much shorter compared to those of LSB galaxies and outer disks of spiral galaxies, so they deviate significantly from the Silk-Elmegreen relation that holds for LSB galaxies and the outer disks of spiral galaxies. In addition, star formation regions in dwarf outer disks show high gas/star mass ratios, ranging from $1: 1$ to $>100: 1$, with a median around 20:1 as shown in Figure 4. The integrated measurements of dwarf outer disks by Elmegreen \& Hunter (2015) confirm the validity of the extended Schmidt law, although their best-fit slope $\left(\Sigma_{\mathrm{SFR}} / \Sigma_{\text {gas }} \propto \sim \Sigma_{\text {star }}^{0.7}\right)$ is a bit steeper than ours $\left(\Sigma_{\mathrm{SFR}} / \Sigma_{\text {gas }} \propto \sim \Sigma_{\text {star }}^{0.6}\right)$. This difference could be due to the different dynamic ranges over which the fits were done, with ours extending into the starburst regime. As shown in Figure 3 and Table 3, the dispersion of outer disk regions is relatively large, which is likely due to large errors (up to 0.5 dex at $1 \sigma$ ) in measuring the SFR, gas mass, and stellar mass in the faint outer disk.

We also investigate the role of the stellar mass at scales of GMCs in M33 with aperture sizes of $70 \mathrm{pc}$. These GMCs also follow the power-law trend. Their overall scatter around the best-fit extended Schmidt law is 0.29 dex, smaller than 0.50 dex around the best-fit Kennicutt-Schmidt law.

To summarize, star formation regions in a variety of galaxy environments, from outer disks to spirals and merging galaxies, from the sub-kiloparsec scale to the GMC scale, from the local to the distant universe, all follow a single power law among SFRs, gas masses, and stellar masses. This may indicate the existence of a single mechanism that drives star formation across different galactic environments.

Throughout the sample, we adopted the same CO conversion factor for all sources instead of using larger values for spirals and smaller values for starburst galaxies. While starburst galaxies on average should have smaller conversion factors than spirals, it is still not well understood under what condition a smaller factor should be used and how much smaller this factor should be (e.g., Narayanan et al. 2012). For spatially resolved individual regions in starburst galaxies, this factor should vary according to the local condition (Pereira-Santaella et al. 2011; Xu et al. 2015). As a result, we used the same factor for simplicity. If adopting smaller values for starburst galaxies, it will steepen the slope of both the Kennicutt-Schmidt law (e.g., Genzel et al. 2010; Liu et al. 2015) and the extended Schmidt law.

\section{Other Outliers of the Kennicutt-Schmidt Law in the Extended Schmidt Plane}

In addition to the low-density regions/galaxies, studies have identified other outliers to the Kennicutt-Schmidt law, suggesting additional parameters in regulating star formation.

Shi et al. (2014) have found that star formation regions of extremely metal-poor galaxies with metallicities below $10 \%$ show significantly lower SFR surface densities in the Kennicutt-Schmidt plane. The SFR measurements are based on the combination of the $24 \mu \mathrm{m}$ and far-UV emission. Since the emission of carbon monoxide in these galaxies is extremely faint (Shi et al. 2015, 2016), the total cold gas masses are obtained through spatially resolved dust and H I maps. We derived the stellar mass using the Spitzer $3.6 \mu \mathrm{m}$ flux of each region available in Shi et al. (2014) using the conversion by Leroy et al. (2008). As shown in Figure 5, the offset of these metal-poor regions is significantly reduced, with the median offset dropping from a factor of 60 in the Kennicutt-Schmidt plane to a factor of 9 in the extended Schmidt plane. 
Table 3

The Median Offsets and the Scatter of Galaxies from the Extended Schmidt, the Kennicutt-Schmidt, and Silk-Elmegreen Laws

\begin{tabular}{lccc}
\hline \hline Galaxy Types & $\begin{array}{c}\text { Extended Schmidt Law } \\
\text { (median, } \sigma \text { ) }\end{array}$ & $\begin{array}{c}\text { Kennicutt-Schmidt Law } \\
\text { (median, } \sigma \text { ) }\end{array}$ & $\begin{array}{c}\text { Silk-Elmegreen Law } \\
(\text { median, } \sigma \text { ) }\end{array}$ \\
\hline Outer disks of dwarfs & $-0.26 \pm 0.04,0.48 \pm 0.03$ & $-1.20 \pm 0.04,0.43 \pm 0.03$ & $-1.92 \pm 0.05,0.31 \pm 0.04$ \\
Local spirals & $-0.01 \pm 0.00,0.24 \pm 0.003$ & $0.02 \pm 0.01,0.33 \pm 0.004$ & $0.03 \pm 0.01,0.39 \pm 0.01$ \\
GMCs in M33 & $0.06 \pm 0.02,0.29 \pm 0.02$ & $0.01 \pm 0.04,0.50 \pm 0.06$ & $\ldots$ \\
Local (U)LIRGs & $0.56 \pm 0.06,0.46 \pm 0.04$ & $0.53 \pm 0.05,0.44 \pm 0.04$ & $\ldots$ \\
High- $z$ MS and SB galaxies & $0.06 \pm 0.02,0.29 \pm 0.02$ & $0.08 \pm 0.05,0.46 \pm 0.04$ & $0.08 \pm 0.06,0.47 \pm 0.07$ \\
\hline
\end{tabular}

Note. The uncertainties of the median and scatter are obtained by the bootstrap.

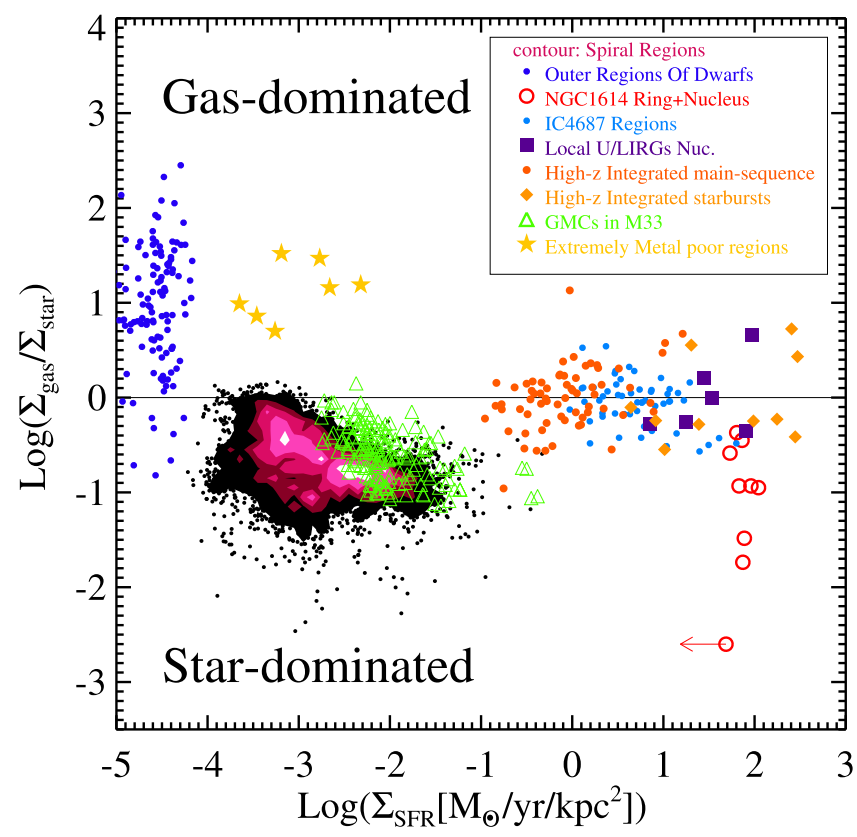

Figure 4. Gas-to-stellar mass ratio as a function of the SFR surface density for our sample.

Roychowdhury (2017) have examined the integrated measurements of metal-poor galaxies and found that they follow the extended Schmidt law better, with a mean offset of 0.01 dex, compared to a mean offset of 0.3 dex in the Kennicutt-Schmidt plane. This indicates that in metal-poor galaxies where the metal plays a crucial role in regulating star formation, the stellar mass should still be important for regulating the star formation processes.

\section{Theoretical Interpretations}

The validity of the extended Schmidt law for a wide range of galactic environments points to the persistent role of the stellar mass in helping to regulate star formation. Star formation can be described as the collapse of a given gas mass over a given timescale, i.e., $\mathrm{SFR}=\eta M_{\mathrm{gas}} / t_{\text {star-formation, }}$, where $\eta$ is a constant describing the fraction of gas mass finally converted into stars. The extended Schmidt law thus implies that the star-forming timescale is governed by the stellar mass, equal to $1 / \Sigma_{\text {star, }}$, which is equivalent to the freefall time for gas in a stellardominated gravitational potential with a constant stellar height (e.g., Shi et al. 2011). Such a phenomenological model, however, does not identify any specific mechanism regarding the role of the stellar mass. Stars can affect the star formation process through stellar radiation pressure, gas heating, and/or stellar gravity. In star-forming regions, the radiation from old stars (the main component of the stellar mass) is usually not important, compared to radiation from young stars.

Stellar gravity is known to play key roles in converting gas into stars. It helps to develop the gravitational instability of cold gas (Jog \& Solomon 1984; Rafikov 2001). As shown by Dib et al. (2017), if the fastest growing mode of the instability is associated with star formation, the observational relationship between SFR, gas, and stars in NGC 628 could be produced from the model. Stellar gravity is also important to the gas pressure. As supported by observations, the pressure is related to the fraction of gas in the molecular phase in which star formation takes place (Elmegreen 1993; Blitz \& Rosolowsky 2006). Additionally, the gas pressure also affects the gas thermal and dynamical status, making star formation environment-dependent, a scenario that has recently been widely explored (Dib et al. 2011; Monaco et al. 2012; Krumholz 2013; Shetty et al. 2013; Meidt 2016). Particularly, in a series of models by Kim et al. (2011), Ostriker et al. (2010), and Ostriker \& Shetty (2011), the stellar mass was quantitatively incorporated into the pressure term to predict the SFRs. It has been proposed that star formation is self-regulated, with SFRs essentially being proportional to the midplane gas pressure that is set by the gravity of gas itself, stars, and dark matter. In spiral disks and outer disks with moderate and low SFRs, the selfregulation is established through balancing the heating by massive stars with the gas cooling that is determined by the gas pressure. In galaxies with high SFRs, the momentum injected into the disk by massive stars is proposed to balance the weight of gas. We measured the midplane gas pressure for our local spirals and outer disks using Equation (35) of Kim et al. (2011):

$$
\begin{aligned}
P_{\text {tot }, \mathrm{DE}}= & 1.7 \times 10^{3} k_{\mathrm{B}} f_{\text {diff }}\left(\frac{\Sigma_{\mathrm{gas}}}{10 M_{\odot} \mathrm{pc}^{-2}}\right)^{2} \\
& \times\left\{\left(2-f_{\text {diff }}\right)+\left[\left(\left(2-f_{\text {diff }}\right)^{2}+37\left(\frac{\sigma_{\mathrm{z}, \mathrm{diff}}}{7 \mathrm{~km} \mathrm{~s}^{-1}}\right)^{2}\right.\right.\right. \\
& \left.\left.\times\left(\frac{\rho_{\mathrm{sd}}}{0.1 M_{\odot} \mathrm{pc}^{-3}}\right)\left(\frac{\Sigma_{\mathrm{gas}}}{10 M_{\odot} \mathrm{pc}^{-2}}\right)^{-2}\right]^{1 / 2}\right\} .
\end{aligned}
$$

Following Kim et al. (2011), $f_{\text {diff }}$ is the mass fraction of the diffuse component $\left(<50 \mathrm{~cm}^{-3}\right)$ and is set to be 0.8 for Figure 6 , $\sigma_{\mathrm{z} \text {,diff }}$ is the total vertical velocity dispersion in the diffuse gas and is set to be $7.0 \mathrm{~km} \mathrm{~s}^{-1}$, and $\rho_{\mathrm{sd}}$ is the midplane density of the stellar disk plus that of the dark matter halo and is set to be $\Sigma_{\text {star }} / h$, where $h$ is the scale height and is set to be $300 \mathrm{pc}$ for 


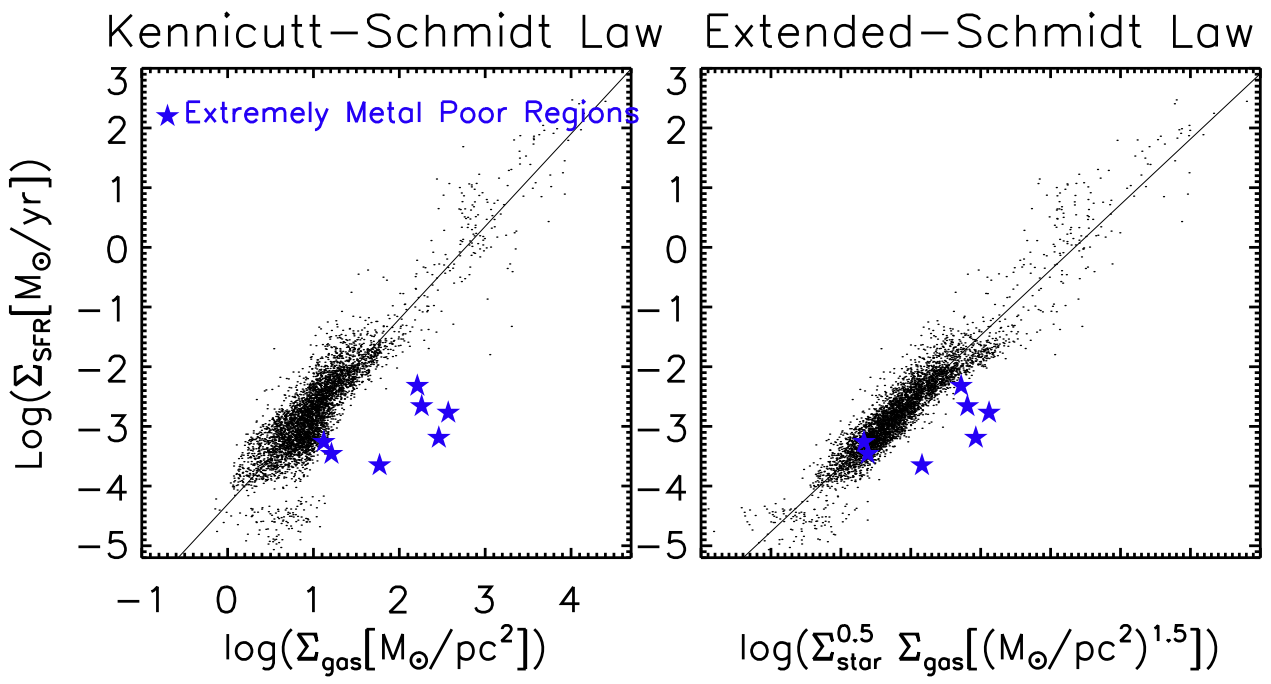

Figure 5. Locations of extremely metal-poor star formation regions on the Kennicutt-Schmidt plane (left panel) and the extended Schmidt plane (right panel).

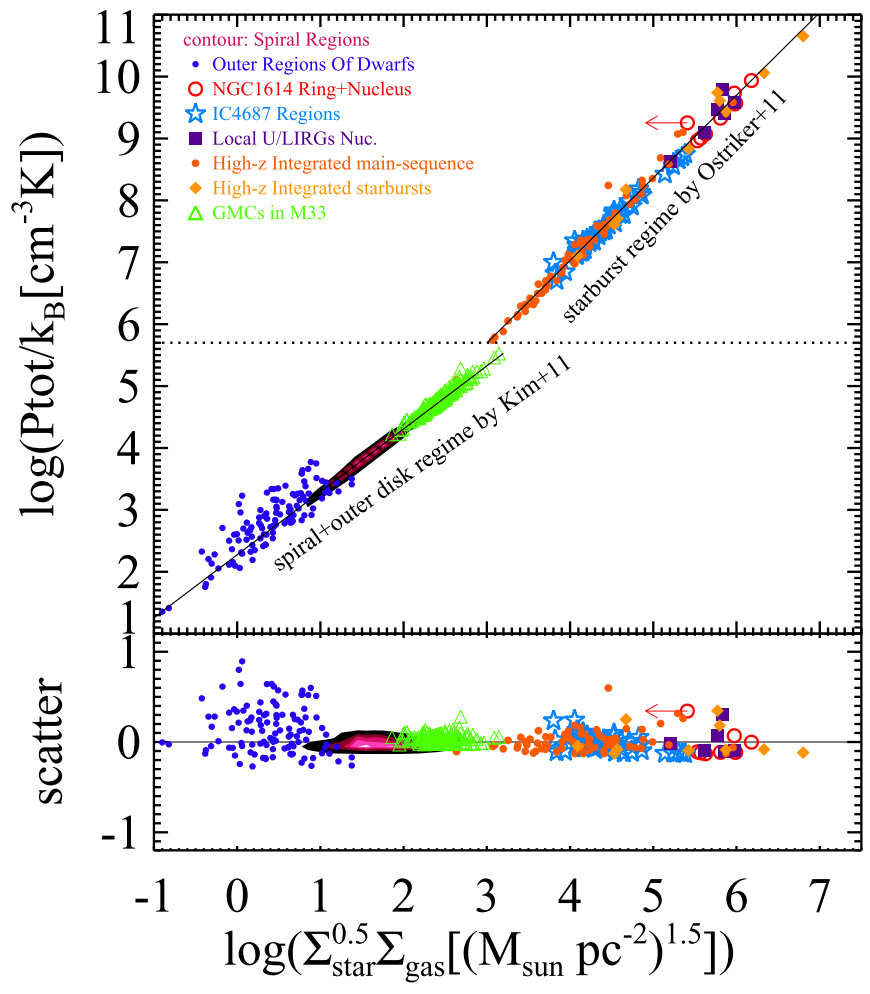

Figure 6. Relationship of the theoretical midplane gas pressure with $\Sigma_{\text {star }}^{0.5} \Sigma_{\text {gas }}$. The two solid lines are the best linear fits to two regimes. The horizontal dotted line is the division between the starburst regime and the disk regime, each of which has a different equation used to estimate the midplane pressure (see the text).

spirals and $1 \mathrm{kpc}$ for the dwarf outer disks. Plugging the $\Sigma_{\text {star }}$ and $\Sigma_{\text {gas }}$ into the above equation, the $P_{\text {tot,DE }}$ is plotted against the term $\Sigma_{\text {star }}^{0.5} \Sigma_{\text {star }}$ in Figure 6.

For mergers and high- $z$ galaxies using Equation (3) of (Ostriker \& Shetty 2011):

$$
P_{\text {eff }}=\frac{\pi G \Sigma_{\text {gas }}^{2}}{2}(1+\chi),
$$

where $\chi=4 \xi_{d} \rho_{\text {bulge }} /\left(3 \rho_{\text {gas }, 0}\right), \xi_{d}$ is the numerical coefficient for the gas vertical distribution but is insensitive to the exact distribution and is set to be 0.33 , as suggested by Ostriker \& Shetty (2011). $\rho_{\text {bulge }}$ is the mass density of the bulge, and $\rho_{\mathrm{gas}, 0}$ is the midplane gas density. We approximate $\rho_{\text {bulge }} / \rho_{\text {gas }, 0}$ by the observed ratio $\Sigma_{\text {star }} / \Sigma_{\text {gas }}$. This is because, for starburst galaxies, we focused on the central regions so that $\Sigma_{\text {star }}$ would represent a measurement of the stellar mass in the bulge.

As shown in Figure 6, in both regimes the midplane pressure is well approximated by the term $\Sigma_{\text {star }}^{0.5} \Sigma_{\text {star. }}$. This suggests that the stellar mass plays a key role in regulating star formation through its gravity.

\section{Conclusions}

The extended Schmidt law suggests a dependence of the star formation efficiency on the stellar mass surface density (Shi et al. 2011). In this study we revisit this relationship by including spatially resolved measurements of SFRs, gas masses, and stellar masses of star formation regions in a vast range of galactic environments, from the outer disks of dwarf galaxies to spiral disks and to merging galaxies, as well as individual molecular clouds in M33. As a result of this study, we find:

(1) Spatially resolved star formation regions are distributed in a tight power-law as $\Sigma_{\mathrm{SFR}} \propto \Sigma_{\text {star }}^{0.5} \Sigma_{\text {gas }}$, which is also valid for the integrated measurements of disk and merging galaxies at high- $z$.

(2) Star formation regions in the outer disks of dwarf galaxies, with $\Sigma_{\mathrm{SFR}}$ down to $10^{-5} M_{\odot} \mathrm{yr}^{-1} \mathrm{kpc}^{-2}$, which are the outliers of both the Kennicutt-Schmidt and SilkElmegreen laws, follow the extended Schmidt law in spite of large scatter caused by photometric errors.

(3) Other outliers in the Kennicutt-Schmidt law, such as extremely metal-poor star formation regions, also show significantly reduced deviation from the extended Schmidt law.

(4) The midplane pressure is related to $\Sigma_{\text {star }}^{0.5} \Sigma_{\text {gas }}$, supporting the idea that existing stars help to regulate current star formation through their gravity. 
We thank the anonymous referee for the helpful and constructive suggestions that improved the quality of the paper, Eve Ostriker for discussions of their model and Pereira-Santaella for providing their measurements of SFRs and gas mass of IC 4687. The work is supported by the National Key R\&D Program of China (No. 2017YFA0402704) and the National Natural Science Foundation of China (NSFC grants 11773013, 11733002, and 11373021) and the Excellent Youth Foundation of the Jiangsu Scientific Committee (BK20150014). K.Q. acknowledges the support from NSFC (11473011 and 11590781). This work is based on observations obtained at the Canada-France-Hawaii Telescope (CFHT), which is operated by the National Research Council of Canada, the Institut National des Sciences de l'Univers of the Centre National de la Recherche Scientifique of France, and the University of Hawaii. The GALEX is a NASA Small Explorer. The mission was developed in cooperation with the Centre National d'Etudes Spatiales of France and the Korean Ministry of Science and Technology. This work is based on observations made with the NASA/ESA Hubble Space Telescope, obtained from the data archive at the Space Telescope Science Institute. STScI is operated by the Association of Universities for Research in Astronomy, Inc. under NASA contract NAS 5-26555. This paper makes use of the following ALMA data: ADS/JAO. ALMA\#2011.0.00182.S. ALMA is a partnership of ESO (representing its member states), NSF (USA) and NINS (Japan), together with NRC (Canada), NSC and ASIAA (Taiwan), and KASI (Republic of Korea), in cooperation with the Republic of Chile. The Joint ALMA Observatory is operated by ESO, AUI/ NRAO, and NAOJ. The National Radio Astronomy Observatory is a facility of the National Science Foundation operated under cooperative agreement by Associated Universities, Inc. The Submillimeter Array is a joint project between the Smithsonian Astrophysical Observatory and the Academia Sinica Institute of Astronomy and Astrophysics and is funded by the Smithsonian Institution and the Academia Sinica. This research has made use of the NASA/IPAC Extra-galactic Database (NED), which is operated by the Jet Propulsion Laboratory, California Institute of Technology, under contract with the National Aeronautics and Space Administration.

\section{ORCID iDs}

Yong Shi ib https://orcid.org/0000-0002-8614-6275 Lin Yan (1) https://orcid.org/0000-0003-1710-9339 Qiusheng Gu (ib https://orcid.org/0000-0002-3890-3729 Keping Qiu (ib https://orcid.org/0000-0002-5093-5088 Sabrina Stierwalt (iD https://orcid.org/0000-0002-2596-8531 Min Fang (1) https://orcid.org/0000-0001-8060-1321 Jingwen Wu (iD https://orcid.org/0000-0001-7808-3756 Xianzhong Zheng (i) https://orcid.org/0000-0003-3728-9912 Zhi-Yu Zhang (i) https://orcid.org/0000-0002-7299-2876 Yu Gao (i) https://orcid.org/0000-0003-0007-2197

\section{References}

Armus, L., Mazzarella, J. M., Evans, A. S., et al. 2009, PASP, 121, 559 Bigiel, F., Leroy, A., Walter, F., et al. 2008, AJ, 136, 2846

Bigiel, F., Leroy, A., Walter, F., et al. 2010, AJ, 140, 1194 Blitz, L., \& Rosolowsky, E. 2006, ApJ, 650, 933

Boquien, M., Lisenfeld, U., Duc, P.-A., et al. 2011, A\&A, 533, A19
Brinchmann, J., Charlot, S., White, S. D. M., et al. 2004, MNRAS, 351, 1151 Capak, P. L., Riechers, D., Scoville, N. Z., et al. 2011, Natur, 470, 233 Carignan, C., \& Beaulieu, S. 1989, ApJ, 347, 760

Carilli, C. L., Hodge, J., Walter, F., et al. 2011, ApJL, 739, L33

Daddi, E., Dannerbauer, H., Krips, M., et al. 2009, ApJL, 695, L176 Dale, D. A., Cohen, S. A., Johnson, L. C., et al. 2009, ApJ, 703, 517 Deul, E. R., \& van der Hulst, J. M. 1987, A\&AS, 67, 509

Dib, S., Hony, S., \& Blanc, G. 2017, arXiv:1702.02609

Dib, S., Piau, L., Mohanty, S., \& Braine, J. 2011, MNRAS, 415, 3439 Elmegreen, B. G. 1993, ApJ, 411, 170

Elmegreen, B. G. 1997, Revista Mexicana de Astronomia y Astrofisica Conference Series, 6, 165

Elmegreen, B. G., \& Hunter, D. A. 2015, ApJ, 805, 145

Genzel, R., Tacconi, L. J., Gracia-Carpio, J., et al. 2010, MNRAS, 407, 2091 Gratier, P., Braine, J., Rodriguez-Fernandez, N. J., et al. 2010, A\&A, 522, A3 Gwyn, S. D. J. 2008, PASP, 120, 212

Hainline, L. J., Blain, A. W., Smail, I., et al. 2011, ApJ, 740, 96

Helfer, T. T., Thornley, M. D., Regan, M. W., et al. 2003, ApJS, 145, 259

Hodge, J. A., Riechers, D., Decarli, R., et al. 2015, ApJL, 798, L18

Holwerda, B. W., Pirzkal, N., de Blok, W. J. G., et al. 2011, MNRAS, 416, 2401

Jog, C. J., \& Solomon, P. M. 1984, ApJ, 276, 127

Kennicutt, R. C., Jr. 1989, ApJ, 344, 685

Kennicutt, R. C., Jr. 1998a, ApJ, 498, 541

Kennicutt, R. C., Jr. 1998b, ARA\&A, 36, 189

Kennicutt, R. C., Jr., Armus, L., Bendo, G., et al. 2003, PASP, 115, 928

Kennicutt, R. C., Jr., Calzetti, D., Walter, F., et al. 2007, ApJ, 671, 333

Kim, C.-G., Kim, W.-T., \& Ostriker, E. C. 2011, ApJ, 743, 25

König, S., Aalto, S., Muller, S., Beswick, R. J., \& Gallagher, J. S. 2013, A\&A, 553, A72

Krumholz, M. R. 2013, MNRAS, 436, 2747

Lanz, L., Ogle, P. M., Alatalo, K., \& Appleton, P. N. 2016, ApJ, 826, 29

Leroy, A. K., Walter, F., Brinks, E., et al. 2008, AJ, 136, 2782

Liu, L., Gao, Y., \& Greve, T. R. 2015, ApJ, 805, 31

Madau, P., \& Dickinson, M. 2014, ARA\&A, 52, 415

Magnier, E. A., \& Cuillandre, J.-C. 2004, PASP, 116, 449

Meidt, S. E. 2016, ApJ, 818, 69

Monaco, P., Murante, G., Borgani, S., \& Dolag, K. 2012, MNRAS, 421, 2485

Morrissey, P., Conrow, T., Barlow, T. A., et al. 2007, ApJS, 173, 682

Murphy, E. J., Bremseth, J., Mason, B. S., et al. 2012, ApJ, 761, 97

Narayanan, D., Krumholz, M. R., Ostriker, E. C., \& Hernquist, L. 2012, MNRAS, 421, 3127

Oh, S.-H., Hunter, D. A., Brinks, E., et al. 2015, AJ, 149, 180

Ostriker, E. C., McKee, C. F., \& Leroy, A. K. 2010, ApJ, 721, 975

Ostriker, E. C., \& Shetty, R. 2011, ApJ, 731, 41

Pereira-Santaella, M., Alonso-Herrero, A., Santos-Lleo, M., et al. 2011, A\&A, 535, A93

Pereira-Santaella, M., Colina, L., García-Burillo, S., et al. 2016, A\&A, 587, A44

Rafikov, R. R. 2001, MNRAS, 323, 445

Riechers, D. A., Bradford, C. M., Clements, D. L., et al. 2013, Natur, 496, 329

Riechers, D. A., Capak, P. L., Carilli, C. L., et al. 2010, ApJL, 720, L131

Riechers, D. A., Carilli, C. L., Capak, P. L., et al. 2014, ApJ, 796, 84

Rieke, G. H., Alonso-Herrero, A., Weiner, B. J., et al. 2009, ApJ, 692, 556

Rogstad, D. H., Wright, M. C. H., \& Lockhart, I. A. 1976, ApJ, 204, 703

Roychowdhury, S., Chengalur, J. N., \& Shi, Y. 2017, A\&A, 608, A24

Roychowdhury, S., Huang, M.-L., Kauffmann, G., Wang, J., \& Chengalur, J. N. 2015, MNRAS, 449, 3700

Schmidt, M. 1959, ApJ, 129, 243

Shetty, R., Kelly, B. C., \& Bigiel, F. 2013, MNRAS, 430, 288

Shi, Y., Armus, L., Helou, G., et al. 2014, Natur, 514, 335

Shi, Y., Helou, G., Yan, L., et al. 2011, ApJ, 733, 87

Shi, Y., Wang, J., Zhang, Z.-Y., et al. 2015, ApJL, 804, L11

Shi, Y., Wang, J., Zhang, Z.-Y., et al. 2016, NatCo, 7, 13789

Silk, J. 1997, ApJ, 481, 703

Tabatabaei, F. S., Beck, R., Krause, M., et al. 2007, A\&A, 466, 509

Tacconi, L. J., Neri, R., Genzel, R., et al. 2013, ApJ, 768, 74

Walter, F., Brinks, E., de Blok, W. J. G., et al. 2008, AJ, 136, 2563

Wilson, C. D., Petitpas, G. R., Iono, D., et al. 2008, ApJS, 178, 189

Wyder, T. K., Martin, D. C., Barlow, T. A., et al. 2009, ApJ, 696, 1834

Xu, C. K., Cao, C., Lu, N., et al. 2015, ApJ, 799, 11

Zibetti, S., Charlot, S., \& Rix, H.-W. 2009, MNRAS, 400, 1181 